\title{
Alterations of aqueous humour outflow following argon laser trabeculoplasty in monkeys
}

\author{
SHLOMO MELAMED AND DAVID L EPSTEIN \\ From the Howe Laboratory of Ophthalmology, Massachusetts Eye and Ear Infirmary, Harvard Medical \\ School, Boston, USA
}

SUMMARY We performed argon laser trabeculoplasty in one eye in each of three cynomolgus monkeys, leaving the contralateral eyes as controls. Four weeks later in both eyes of each monkey we infused cationised ferritin as a tracer of the aqueous outflow pathways for 30-40 minutes before fixation. In the control eyes cationised ferritin was found throughout the conventional aqueous outflow tract, labelling trabecular endothelial cells, both inner and outer walls of Schlemm's canal, and the collecting channels. No permeation of cationised ferritin through continuous cellular layers such as the corneal endothelium was detected. In the lasered spots of the treated eyes cationised ferritin was found to label the cellular sheet covering the trabecular scar at the lasered site, but no tracer was detected within the scar or in Schlemm's canal immediately beneath. In contrast in adjacent non-lasered regions the trabecular cells, the observed juxtacanalicular herniations, and the vacuoles of the inner wall as well as Schlemm's canal itself were extensively labelled. Our findings suggest impermeability to aqueous humour flow through the actual lasered lesion, with shift of flow to adjacent, non-lasered regions. In the normal monkey these regions compensate structurally and functionally in ways that may help to deal with this diverted flow.

Short term ${ }^{1-3}$ and long term effects ${ }^{2-5}$ of argon laser trabeculoplasty have already been described by us and others. Four weeks following argon laser trabeculoplasty in monkeys we have observed that the lasered sites contain flattened scarred trabecular meshwork with a cellular extension over the scar and with a flat inner wall of Schlemm's canal beneath. ${ }^{6}$ Adjacent non-lasered regions were observed to have wide open intertrabecular spaces, with herniations of juxtacanalicular trabecular meshwork and the inner wall into and across the lumen of Schlemm's canal.

In order to study possible regional changes in aqueous outflow associated with these morphological changes we performed a tracer study using cationised ferritin in vivo four weeks after argon laser trabeculoplasty. This positively charged ferritin (MW 800000 ) developed by Dannon et al. ${ }^{7}$ binds to negatively charged surfaces such as those that occur on a cellular membrane. This binding prevents the washout of the tracer during the fixation process and allows the delineation of the outflow routes. We have pre-

Correspondence to Dr S Melamed, Howe Laboratory of Ophthalmology, Massachusetts Eye and Ear Infirmary, 243 Charles Street, Boston, MA 02114, USA. viously demonstrated that cationised ferritin can be used effectively as an ultrastructural tracer of aqueous flow patterns in the trabecular meshwork of the living monkey. ${ }^{8}$

\section{Material and methods}

Three sexually mature cynomolgus monkeys were used for this study. Argon laser trabeculoplasty was performed in the right eye of each with an energy setting of $400-700 \mathrm{MW}$, as described previously, ${ }^{1}$ while the left eye remained untreated and served as a control. Intraocular pressures were determined with a Digilab pneumotonometer prior to and four weeks after laser treatment. Four weeks after argon laser trabeculoplasty the monkeys were anaesthetised with ketamine hydrochloride intramuscularly $(10 \mathrm{mg} / \mathrm{kg})$ followed by sodium pentobarbital intravenously ( 35 $\mathrm{mg} / \mathrm{kg}) .2 \mathrm{ml}(10.4 \mathrm{mg})$ of cationised ferritin in isotonic solution (measured $\mathrm{pH}=6 \cdot 18$, Sigma Inc., St Louis) was perfused into the anterior chamber as follows. The cationised ferritin was introduced through a 25 gauge needle into the anterior chamber from a reservoir at a height of $17 \mathrm{mmHg}$, while 


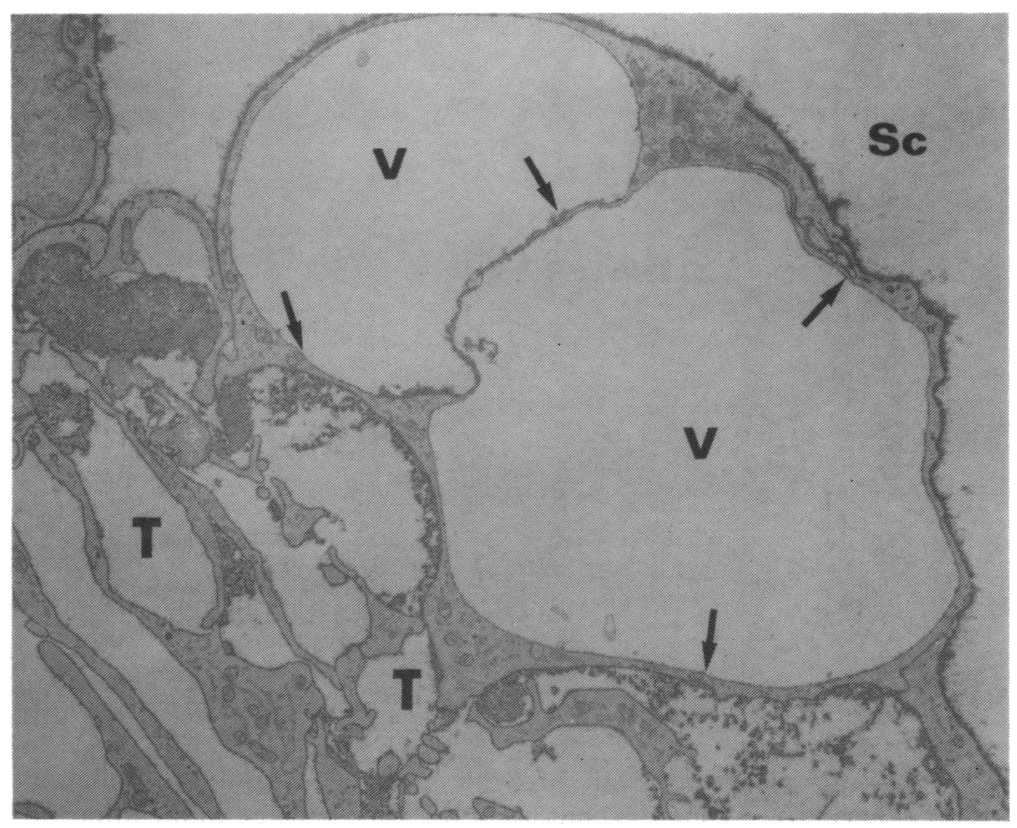

Fig. 1 Control eye. Cationised ferritin labels cellular membranes along the trabecular spaces $(\mathrm{T})$ and inner wall of Schlemm's canal (Sc). Note uniform labelling of inner membranes (arrows) of giant vacuoles $(\mathrm{V})$. TEM. $\times 6425$.

another reservoir containing Dulbecco's phosphate buffered saline with $5.5 \mathrm{mM}$ added glucose was connected through a separate 25 gauge needle to the anterior chamber at a height of $13 \mathrm{mmHg}$. After 30-40 minutes of flow of ferritin through the anterior chamber from one reservoir to the other the egress route was clamped, and $3 \%$ glutaraldehyde in $0.1 \mathrm{M}$ cacodylate buffer was infused into the anterior chamber for one hour at a pressure of $15 \mathrm{mmHg}$. The monkeys were then killed by an intravenous overdose of pentobarbital, and the fixative perfusion was continued for 20 minutes. The eyes were then enucleated, the anterior segments removed, and meridional sections of the iridocorneal angle were prepared. These tissue sections were postfixed in $1 \%$ osmium tetroxide with $1.5 \%$ potassium ferrocyanide, passed through graded alcohol concentrations, and embedded in Epon-Araldite. Thin sections were made $(50 \mathrm{~nm}$, silver-golden), stained with uranyl acetate and lead citrate, and observed with the JEOL JEM 7 electron microscope.

\section{Results}

Four weeks after argon laser trabeculoplasty there was no change of intraocular pressure in the treated eyes (mean of $18 \mathrm{mmHg}$ compared with mean of 17 $\mathrm{mmHg}$ in the control eyes).

In the control eyes cationised ferritin was found throughout the outflow tract. There was labelling along cell membranes of the trabecular endothelial

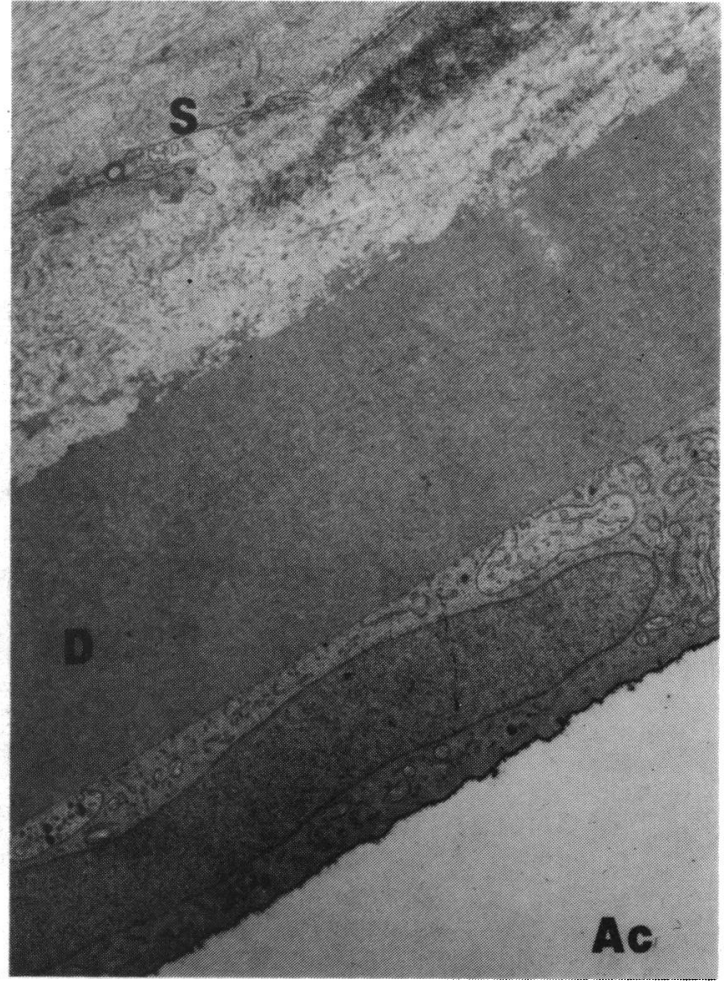

Fig. 2 Control eye. Corneal endothelium. Cationised ferritin labels the cellular membrane facing the anterior chamber (AC), but no tracer is detected in Descemet's membrane (D) or corneal stroma (C) beneath. TEM. $\times 4410$. 
Fig. 3 Control eye. Collector channel. Note the thick layer of cationised ferritin labelling the luminal side of the endothelium (E), but no tracer is seen to permeate into the adjacent sclera (S). TEM. $\times 9695$ :

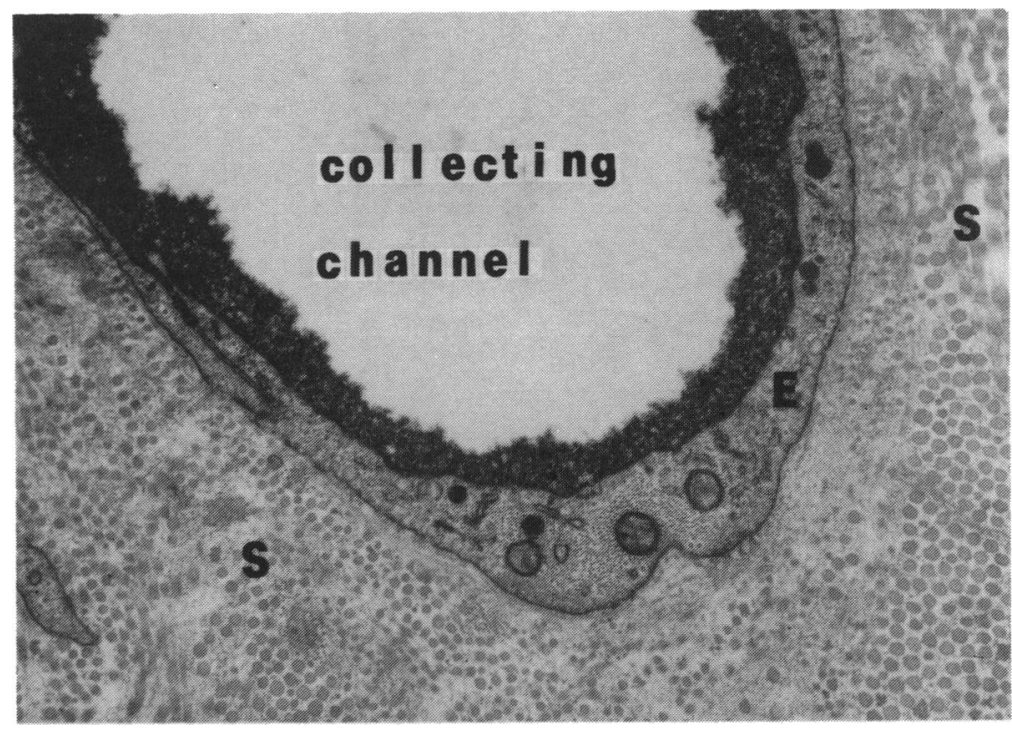

cells, juxtacanalicular cells, the inner membranes of vacuoles, and the luminal side of the inner wall of Schlemm's canal (Fig. 1). Uniform labelling of the corneal endothelium was also evident, but no tracer was detected in Descemet's membrane or the corneal stroma (Fig. 2). A thick layer of cationised ferritin particles was found to label the endothelium of the outer wall of Schlemm's canal and the collector channels, but no tracer was detected in the adjacent sclera (Fig. 3). In the eyes treated with argon laser trabeculoplasty lasered spots appeared as scarred trabecular meshwork, with extension of corneal endothelium over the flattened, collapsed beams and flat inner wall of Schlemm's canal. Cationised ferritin

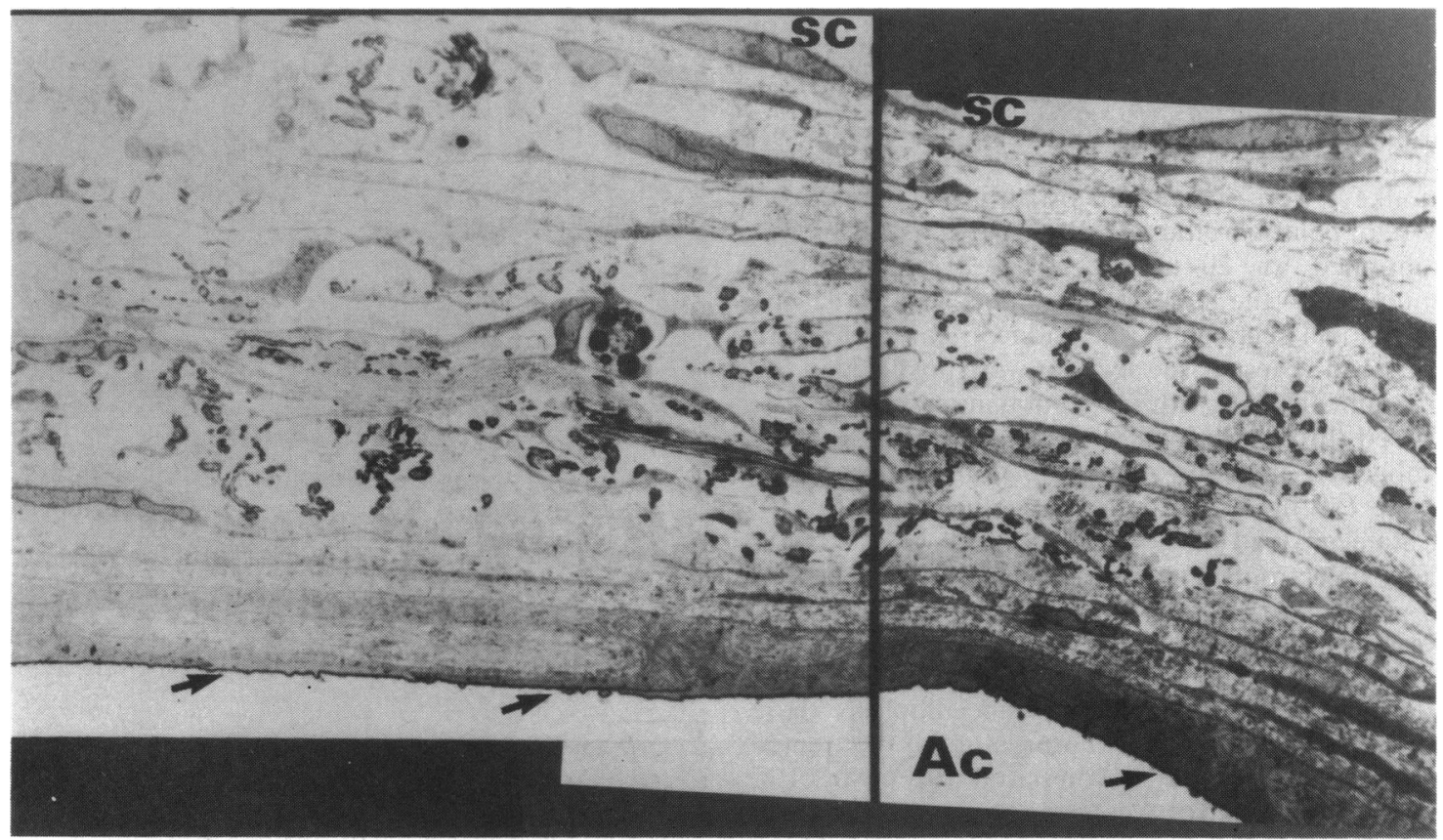

Fig. 4 Lasered spot, four weeks after argon laser trabeculoplasty. The trabecular meshwork is flattened and scarred.

Cationised ferritin labels the cellular sheet extending from the corneal endothelium (arrows). No tracer is detected in the scar under this cellular cover nor on the inner wall of Schlemm's canal (Sc). AC=anterior chamber. TEM. $\times 1055$. 


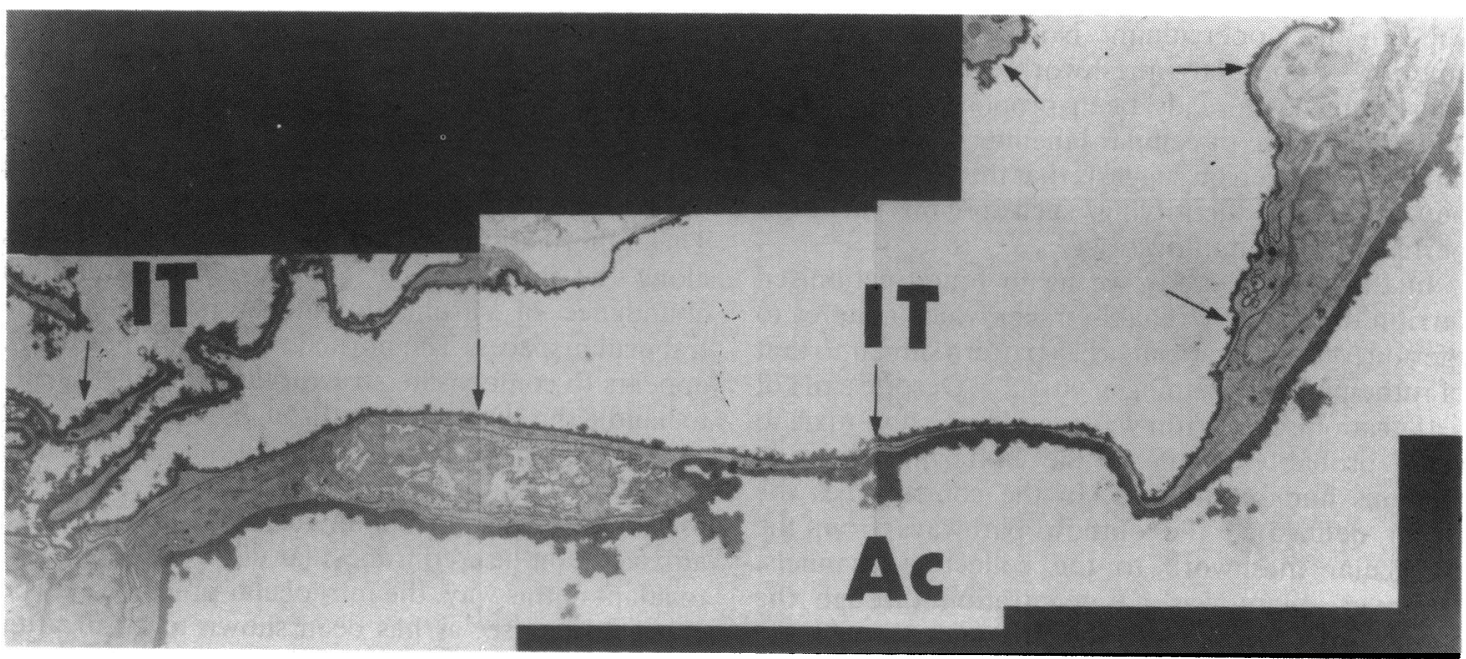

Fig. 5 Non-lasered region of eye treated with argon laser trabeculoplasty. Intertrabecular spaces (IT) are wide open and cationised ferritin labels trabecular endothelial cells on the beams (arrows). TEM composite. $\times 2015$.

labelled the cellular sheet, but no tracer could be detected within the scar or on the luminal surface of the inner wall of Schlemm's canal distal to the laser lesion (Fig. 4). In adjacent non-lasered regions the

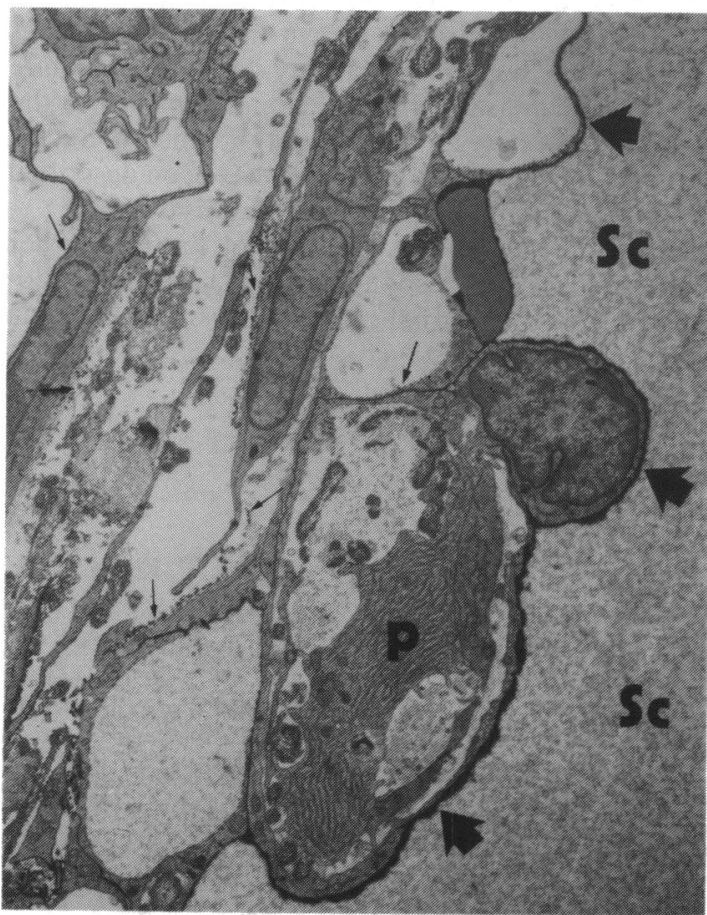

Fig. 6 Non-lasered region of argon laser trabeculoplasty treated eye. Herniation of juxtacanalicular meshwork (arrows). Note the uniform thick labelling with cationised ferritin (arrowheads) of the inner wall of Schlemm's canal (Sc). $\mathrm{P}=$ probably a plasma cell. TEM. $\times 1765$. intertrabecular spaces were wide open, with uniform labelling of trabecular endothelial cells lying over the beams (Fig. 5). Herniations of juxtacanalicular trabecular meshwork into Schlemm's canal were stained throughout their protrusions, with extensive labelling of the inner wall of Schlemm's canal (Fig. 6).

\section{Discussion}

In this study we searched for a correlation between the observed morphological changes and alterations of aqueous outflow within the angle of the anterior chamber following argon laser trabeculoplasty. For delineating the outflow routes, numerous tracers such as latex spheres, ${ }^{9-11}$ thorium dioxide,,$^{91213}$ and anionic ferritin particles ${ }^{14} 15$ have been used in the past. However, the main obstacle to the precise morphological description of the outflow pathways with those tracer particles is their tendency to wash out during perfusion with fixative. To overcome this problem we have used cationised ferritin for perfusion in vivo. ${ }^{8}$ Cationised ferritin is a positively charged molecule which binds to negatively charged surfaces such as cell membranes, a property which makes it capable of adhering strongly to the aqueous outflow routes, thus minimising washout effects. This tracer has already been used for labelling complex carbohydrates of the surface of the cornea in vitro ${ }^{16-18}$ and for labelling endothelial cells of iris vessels by perfusion or intravascular injection in vivo. ${ }^{15} \mathrm{We}$ have previously described a uniform labelling with cationised ferritin of the conventional aqueous outflow tract of the monkey. ${ }^{8}$ Interestingly, however, no tracer could be detected in the trabecular spaces 
beneath the operculum. Non-perfusion of the anterior trabecular meshwork underlying the operculum has already been proposed by others, ${ }^{18}$ and the absence of cellular labelling with cationised ferritin in this region suggests that this technique may be useful for identifying such regions of nonperfusion in the outflow tract.

In the present study we again found cationised ferritin to be a very reliable tracer which adheres to the aqueous outflow routes in a pattern similar to that of ruthenium red staining in vitro. ${ }^{1-21}$ Despite a $\mathrm{pH}$ of $6 \cdot 18$, cationised ferritin was found to be non-toxic to cells, probably owing to the buffering effect of aqueous humour in vivo. In the control eyes the tracer delineated the outflow pathways from the trabecular meshwork to the collecting channels. However, there was no penetration through the corneal endothelium into the stroma or through the endothelial lining of the outer wall of Schlemm's canal or the collecting channels into adjacent sclera, suggesting that cationised ferritin follows the route for the bulk outflow of aqueous humour.

In the laser treated eye's scarred lasered spots were impermeable to cationised ferritin, which labelled only the cellular sheet covering the lesion. Within the time frame of the perfusion there was no staining of Schlemm's canal beneath the scar, indicating an absence of distal flow. However, the adjacent nonlasered areas were labelled throughout the outflow tract, including the lumen of Schlemm's canal, suggesting a shift of flow from the scarred lasered sites to the non-lasered regions. We propose, therefore, that in these non-glaucomatous monkeys, four weeks after argon laser trabeculoplasty the lasered scars of the trabecular meshwork are impermeable to aqueous humour, which then must shift to the path of less resistance through the adjacent non-lasered trabecular regions. In a preceding report ${ }^{6}$ we demonstrated herniations of juxtacanalicular meshwork into and across the lumen of Schlemm's canal in the non-lasered regions of the trabecular meshwork. These herniations contained numerous vacuoles along the inner wall of Schlemm's canal and an abundance of chronic inflammatory cells in the trabecular spaces. The normal trabecular meshwork appears to compensate structurally and functionally to handle this diverted flow through the development of these extensive herniations and the formation of vacuoles, which probably provide an increased area for drainage into Schlemm's canal (Fig. 7). If the adjacent non-lasered trabecular meshwork is unable to adapt in this way, the intraocular pressure may be expected to rise, as has been shown to occur after more extensive confluent laser treatment of the trabecular meshwork in monkeys. ${ }^{22} 23$

It is possible that stimulation of the trabecular cells such as increased turnover of extracellular matrix ${ }^{24}$ or increased phagocytic activity, ${ }^{1}$ which is triggered by argon laser treatment, may have a role in the efficacy of this procedure by removing 'clogging' debris from intertrabecular spaces. However, a decrease in trabecular cellularity, as shown in enucleated eyes after argon laser trabeculoplasty, ${ }^{24}$ might contribute to a late decrease in the functional capacity of the trabecular meshwork and is of major concern. Such a loss of trabecular meshwork cells has been described in aging eyes ${ }^{25}$ and also in glaucomatous eyes. ${ }^{2627}$

From our studies we still do not know whether glaucomatous eyes respond to argon laser trabeculoplasty as do the normal monkey eyes. In glaucoma
Fig. 7 Semischematic illustration of structural changes and aqueous flow alterations following argon laser trabeculoplasty in a normal monkey.

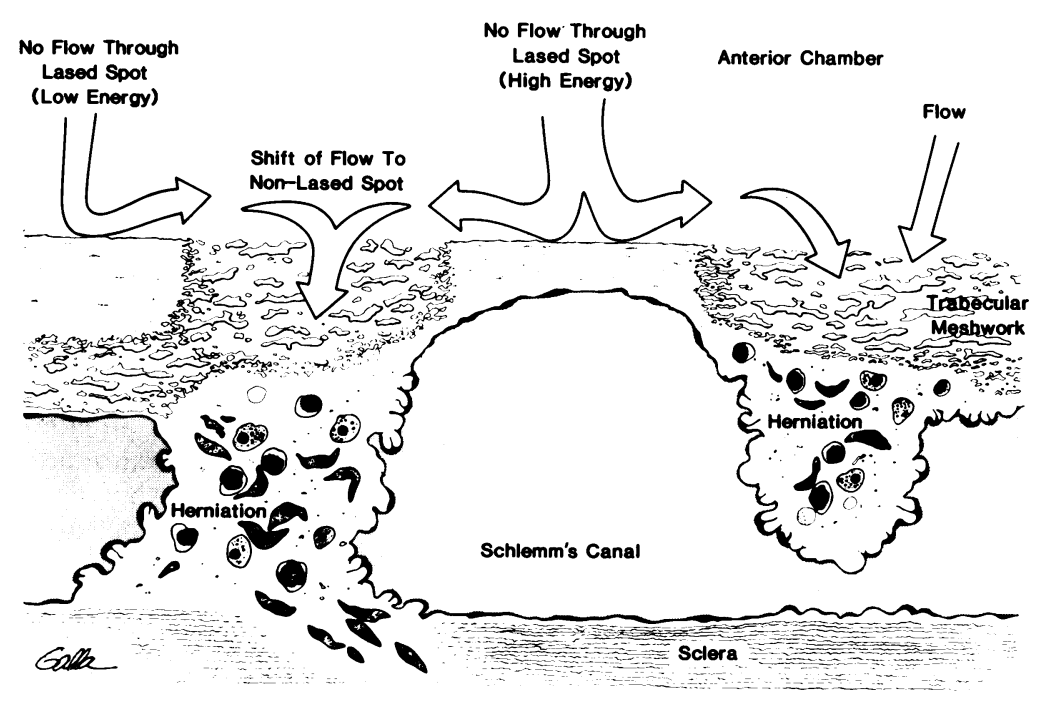


this treatment, when successful, results in intraocular pressure reduction even below prelasered level. If in the glaucomatous human eye the response of the trabecular tissue to argon laser is similar to what we have observed in the monkey, then it is also possible the herniations might be involved in some other biological process that we do not yet understand. Although mechanical shrinkage of collapsed beams may play a part in increasing outflow facility in glaucomatous eyes, ${ }^{28}$ the biological effects and structural alterations that we observed may also be important.

In summary, we suggest that the morphological changes in the trabecular meshwork seen after argon laser trabeculoplasty are associated with regional changes in aqueous outflow. The end result in the normal monkey is to maintain the intraocular pressure unchanged despite the scarring of a substantial portion of the angle tissue. Whether these biological and morphological effects of argon laser trabeculoplasty play a part clinically in intraocular pressure reduction is still unclear, and studies of glaucomatous human eyes are needed.

This study was supported in part by grant EYO1894 and from the National Eye Institute, and a grant from National Glaucoma Research, a programme of American Health Assistance Foundation.

\section{References}

1 Melamed S, Pei J, Epstein DL. Short term effect of argon laser trabeculoplasty in monkeys. Arch Ophthalmol 1985; 103: 1546-52.

2 Rodrigues MM, Spaeth GL, Donohoo P. Electron microscopy of argon laser therapy in phakic open angle glaucoma. Ophthalmology 1985; 89: 198-210.

3 Koss MK, March WF, Nordquist RE, et al. Acute intraocular pressure elevation produced by argon laser trabeculoplasty in the cynomolgus monkey. Arch Ophthalmol 1984; 102: 1699-703.

4 Weber PA, Davidorf FH, McDonald C. Scanning electron microscopy of argon laser trabecularplasty. Ophthalmic Forum 1983; 1: 26-9.

5 Sarita RJ, Fellman RL, Rodrigues MM, Spaeth GL. Histopathologic verification of position of laser burns in argon laser trabeculoplasty. Ophthalmic Surg 1984; 15: 854-9.

6 Melamed S, Pei J, Epstein DL. Delayed response to argon laser trabeculoplasty in monkeys: I. Morphological study and morphometric analysis. Arch Ophthalmol in press.

7 Dannon D, Goldstein L, Marikovsky Y, Skutelsky E. Use of cationized ferritin as a label of negative charges on cell surfaces. J Ultrast Res 1972; 38: 500-10.

8 Melamed S, Freddo FT, Epstein DL. Use of cationized ferritin to trace aqueous humor outflow in the monkey eye. Exp Eye Res in press.
9 Inomata H, Bill A, Smesler GK. Aqueous humor pathways through the trabecular meshwork and into Schlemm's canal in the cynomolgus monkey. An electron microscopic study. Am J Ophthalmol 1972; 73: 760-89.

10 Karg SJ, Garron LK,' Feeney ML, McEwen WK. Perfusion of human eyes with latex microspheres. Arch Ophthalmol 1959; 61: 68-71.

11 Samuelson PA, Gelatt KN, Gum LL. Kinetics of phagocytosis in the normal canine iridocorneal angle. Am J Vet Res 1984; 45: 2359-66.

12 Tripathi RC. The mechanism of aqueous outflow in lower mammals. Exp Eye Res 1972; 14: 73-9.

13 Tripathi RC. The mechanism of aqueous outflow in birds. An ultrastructural study of perfused eyes. Exp Eye Res 1973; 15: 425-34.

14 Feeney L. Symposium-contributions of electron microscopy to the understanding of the production and outflow of aqueous humor outflow studies using an electron dense tracer. Ophthalmology 1966; 70: 791-8.

15 Segawa K. Pores of the trabecular wall of Schlemm's canal. Ferritin perfusion in enucleated human eyes. Nippon Ganka Gakkai Zasshi 1970; 74: 1240-74.

16 Wells PA, Hazlett LD. Complex carbohydrates at the ocular surface of a mouse: an ultrastructural and cytochemical analysis. Exp Eye Res 1984; 39: 19-35.

17 Raviola G, Butler JM. Asymmetric distribution of charged domains on the two fronts of the endothelium of iris blood vessels. Invest Ophthalmol Vis Sci 1985; 26: 597-608.

18 Lutjen-Drecoll E, Kaufman PL, Barrany EH. Light and electron microscopy of the anterior chamber angle structures following surgical disinsertion of the cilary body muscle in the cynomolgus monkey. Invest Ophthalmol Vis Sci 1977; 16: 218-25.

19 Armaly MF, Wang Y. Demonstration of acid mucopolysaccharides in the trabecular meshwork of the rhesus monkey. Invest Ophthalmol Vis Sci 1975; 14: 507-16.

20 Richardson TM. Distribution of glycosaminoglycans in the aqueous outflow system of the cat. Invest Ophthalmol Vis Sci 1982; 22: 319-28.

21 Grierson I, Lee WR, Abraham S. The appearance of the outflow apparatus of the eye after staining with ruthenium red. Acta Ophthalmol (Kbh) 1977; 55: 827-36.

22 Gaasterland D, Kupfer C. Experimental glaucoma in the rhesus monkey. Invest Ophthalmol Vis Sci 1985; 25 (suppl): 158.

23 Quigley HA, Hohman RM. Laser energy levels for trabecular meshwork damage in the primate eye. Invest Ophthalmol Vis Sci 1983; 24 (suppl): 1305-7.

24 Van Buskirk EM, Pond V, Rosenquist RC, Acott TS. Argon laser trabeculoplasty-studies on mechanism of action. Ophthalmology 1984; 91: 1005-10.

25 Alvarado J, Murphy C, Polansky J, Juster R. Age related change in trabecular meshwork cellularity. Invest Ophthalmol Vis Sci $1981 ; 21: 714-27$.

26 Alvarado J, Murphy C, Juster R. Trabecular meshwork cellularity in primary open angle glaucoma and non-glaucomatous normals. Ophthalmology 1984; 91: 564-79.

27 Murphy C, Maglio M, Alvarado J. Morphometric analysis of trabecular meshwork in primary open angle glaucoma: Invest Ophthalmol Vis Sci 1983; 24 (suppl): 135.

28 Wise JB, Witter SC. Argon laser therapy for open angle glaucoma. Arch Ophthalmol 1979; 97: 319-22.

Accepted for publication 6 October 1986. 